\title{
DELIVERY OF THE INSULIN GENE USING AN INTEGRATING ADENO-ASSOCIATED VIRAL VECTOR (AAV) TO DIABETIC NOD MICE
}

Que T. La ${ }^{1}$, Binhai Ren ${ }^{1}$, Grant Logan², Sharon Cunningham², Neeta Khandekar², Najah T. Nassif ${ }^{1}$, Bronwyn A. O'Brien ${ }^{1}$, Ian E. Alexander ${ }^{2,3}$, Ann M. Simpson ${ }^{1}$

${ }^{1}$ School of Life Sciences, University of Technology Sydney, Sydney, Australia

${ }^{2}$ The Children's Hospital at Westmead and Children's Medical Research Institute, Westmead, Sydney, Australia

${ }^{3}$ Discipline of Child and Adolescent Health, University of Sydney, Westmead, Sydney, Australia.

Previously we introduced furin-cleavable human insulin (INS-FUR) to the livers in several animal models using a lentiviral vector. Reversal of diabetes with normal glucose tolerance and pancreatic transdifferentiation was seen. The aim of the present study was to ascertain if we could similarly reverse diabetes in the non-obese diabetic (NOD) mouse using a clinically-applicable AAV system to deliver INS-FUR \pm the $\beta$-cell transcription factor (TF) $P d x 1$ to the livers of the diabetic mice. The traditional AAV8 that allows predominantly episomal expression, and the hybrid AAV8/piggyBac that results in transgene integration into the host genome were used. Weight and blood glucose levels were monitored. Intraperitoneal glucose tolerance tests (IPGTTs) were performed. Immunofluorescence was carried out to monitor transduction efficiency utilising marker genes Venus and mCherry. RT-PCR was used to identify expression of pancreatic genes. Diabetic mice that received IP injections of AAV8-INSFUR-mCherry $\left(2.3 \times 10^{10}-4.5 \times 10^{10} \mathrm{Vg}\right)$ became hypoglycaemic with abnormal responses to IPGTTs. No $\beta$-cell TF could be detected in the livers. Reversal of diabetes could not be achieved in the mice $(n=8)$ that received combinations of AAV8-INSFUR-venus $\left(5 \times 10^{10}\right.$ or $\left.7.5 \times 10^{10} \mathrm{Vg}\right)$ and AAV8-Pdx1-mCherry $\left(7.5 \times 10^{10} \mathrm{Vg}\right)$. Both INS-FUR and $P d \times 1$ were detected using RT-PCR and immunofluorescence showed the expression of both marker genes. IPGTTs were abnormal; however, the $\beta$-cell TF MafA was detected by RT-PCR. Euglycaemia and normal IPGTTs were achieved in mice $(n=4)$ that received AAV8/piggyBac-INS-FUR-mCherry (Transposon $3.5 \times 10^{10} \mathrm{Vg}$ and transposase $4 \times 10^{10}$ $\mathrm{Vg}$ ). The INS-FUR gene and the $\beta$-cell TFs NKX6.1 and MafA were detected in the livers, however wider evidence of pancreatic transdifferentiation was not seen. This is the first in vivo study using the hybrid AAV8/piggyBac system to treat Type 1 diabetes (T1D). The AAV system, with further development, may hold promise for gene therapy of T1D. 